\title{
Statistical approximation by some positive linear operators of discrete type
}

\author{
C. Radu
}




\title{
STATISTICAL APPROXIMATION BY SOME POSITIVE LINEAR OPERATORS OF DISCRETE TYPE
}

\author{
C. RADU
}

Received 13 September, 2007

\begin{abstract}
The aim of this paper is to present a class of linear positive operators of discrete type and its statistical approximation properties obtained by using a Bohman-Korovkin type theorem.

2000 Mathematics Subject Classification: 40A25, 41A36

Keywords: $A$-statistical convergence, Bohman-Korovkin type theorem, sequence of positive linear operators
\end{abstract}

\section{INTRODUCTION}

The study of the statistical convergence for sequences of positive linear operators was attempted in the year 2002 by A. G. Gadjiev and C. Orhan [8]. The research orientation was proved to be extremely fertile, many researchers approaching this subject recently [2-4]. Motivated by this research direction, we construct a general class of positive linear operators of discrete type and study its statistical approximation properties.

In order to construct the operators, we need some notation on $A$-statistical convergence. Let $A:=\left(a_{k n}\right)_{k, n \in \mathbb{N}}$ be a non-negative regular summability matrix. For a given sequence of real numbers, $x:=\left(x_{n}\right)_{n \in \mathbb{N}}$, the sequence $A x:=\left((A x)_{k}\right)$ defined by the formula

$$
(A x)_{k}:=\sum_{n=1}^{\infty} a_{k n} x_{n}
$$

is called the $A$-transform of $x$ whenever the series converges for each $k \in \mathbb{N}$. A sequence $x$ is said to be $A$-statistically convergent to a real number $L$ if for every $\varepsilon>0$, one has

$$
\lim _{k} \sum_{n:\left|x_{n}-L\right| \geq \varepsilon} a_{k n}=0
$$

We denote this limit by $\mathrm{st}_{A}-\lim x=L$ (see [6]). 


\section{DEFINITION OF OPERATORS}

We set $\mathbb{R}_{+}:=[0, \infty)$ and $\mathbb{N}_{0}:=\{0\} \cup \mathbb{N}$. Let $D$ be a given interval of the real line. We denote by $C(D)$ the space of all real-valued continuous functions on $D$. For each $n \in \mathbb{N}$ we consider a set of indices $I_{n}$ and a net on $D$ called $\left(x_{n, j}\right)_{j \in I_{n}}$. We set $e_{i}(x)=x^{i}, i \geq 0, x \in D$. Following [1], let $\left(l_{n}\right)_{n \geq 1}$ be a sequence of positive linear operators of discrete type, defined by the equality

$$
\left(l_{n} f\right)(x)=\sum_{j \in I_{n}} u_{n, j}(x) f\left(x_{n, j}\right), \quad x \in D, f \in C(D),
$$

where $\left(u_{n, j}\right)_{j \in I_{n}}$ is a family of continuous functions on $D$ satisfying the following conditions

$$
\begin{gathered}
u_{n, j}(x) \geq 0, \quad x \in D, \\
\sum_{j \in I_{n}} u_{n, j}(x)=e_{0}(x), \quad x \in D, \\
\sum_{j \in I_{n}} u_{n, j}(x) x_{n, j}=e_{1}(x), \quad x \in D, \\
\sum_{j \in I_{n}} u_{n, j}(x) x_{n, j}^{2}=e_{2}(x)+\psi_{n}(x), \quad x \in D,
\end{gathered}
$$

where $\psi_{n} \in C(D)$.

Under this assumptions the sequence $\left(l_{n}\right)_{n \geq 1}$ can be indicated by the following system

$$
l_{n}:\left\langle D, I_{n}, x_{n, j}, u_{n, j}(x) ; \psi_{n}\right\rangle, \quad(n, j) \in \mathbb{N} \times I_{n}, x \in D .
$$

We denote by $C_{B}(D)$ the space of all continuous functions on $D$ and bounded on the entire line, i. e.,

$$
|f(x)| \leq M_{f} \quad \text { for all } x \in \mathbb{R},
$$

where $M_{f}$ is a constant depending on $f . C_{B}(D)$ is a Banach space with respect to the supremal norm $\|\cdot\|$.

In [1], compounding two sequences of operators given by (2.6), the author constructed a sequence of positive linear operators $\left(L_{n, \lambda}\right)_{n \geq 1}$ acting on $C\left(\mathbb{R}_{+}\right)$.

In what follows, we will replace the conditions (2.3)-(2.5) imposed on the sequence $\left(u_{n, j}\right)_{j \in I_{n}}$ by the following ones:

$$
\begin{gathered}
\mathrm{st}_{A}-\lim _{n}\left\|\sum_{j \in I_{n}} u_{n, j}-e_{0}\right\|=0, \\
\mathrm{st}_{A}-\lim _{n}\left\|\sum_{j \in I_{n}} u_{n, j} x_{n, j}-e_{1}\right\|=0,
\end{gathered}
$$




$$
\mathrm{st}_{A}-\lim _{n}\left\|\sum_{j \in I_{n}} u_{n, j} x_{n, j}^{2}-e_{2}\right\|=0 .
$$

A sequence of positive linear operators of the form (2.1) which satisfies the conditions (2.2) and (2.7)-(2.9) will be denoted by

$$
\tilde{l}_{n}:\left\langle D, I_{n}, x_{n, j}, u_{n, j}(x)\right\rangle, \quad(n, j) \in \mathbb{N} \times I_{n}, x \in D .
$$

Further on we will consider two sequences of operators of the type (2.10) and (2.6), respectively,

$$
\tilde{l}_{n}:\left\langle[0,1], I_{n}, x_{n, j}, u_{n, j}(x)\right\rangle, \quad(n, j) \in \mathbb{N} \times I_{n}, x \in[0,1],
$$

and

$$
l_{n}:\left\langle[0, b], J_{n}, y_{n, j}, v_{n, j}(x) ; \psi_{n}\right\rangle, \quad(n, j) \in \mathbb{N} \times J_{n}, x \in[0, b],
$$

such that $1 \leq b$ and, for any $n \in \mathbb{N}$, there is a function $\widetilde{\psi}_{n} \in C([0, b])$ with the property

$$
x_{n, j} \psi_{j}(x)=\tilde{\psi}_{n}(x), \quad j \in I_{n}, x \in[0, b] .
$$

Let us consider a continuous function $\lambda:[0, b] \rightarrow[0,1]$. Now we are ready to introduce the operator $\widetilde{L}_{n, \lambda}$ by putting

$$
\left(\widetilde{L}_{n, \lambda} f\right)(x)=\sum_{j \in I_{n}} \sum_{s \in J_{j}} u_{n, j}(\lambda(x)) v_{j, s}(x) f\left(x_{n, j} y_{j, s}+\left(1-x_{n, j}\right) x\right)
$$

for all $x \in[0, b], f \in C_{B}([0, b])$, and $n \in \mathbb{N}$. We observe that these operators are positive and linear.

\section{A BOHMAN-KOROVKIN TYPE THEOREM}

In [8], Gadjiev and Orhan proved the following Bohman-Korovkin type statistical approximation theorem.

Theorem A. If a sequence of positive linear operators $A_{n}: C_{B}([a, b]) \rightarrow B([a, b])$ satisfies the conditions

$$
\mathrm{st}-\lim _{n}\left\|A_{n} e_{i}-e_{i}\right\|=0 \quad \text { fori }=0,1,2,
$$

then, for any function $f \in C_{B}([a, b])$, we have

$$
\text { st }-\lim _{n}\left\|A_{n} f-f\right\|=0,
$$

where $B([a, b])$ is the space of all real-valued functions bounded on $[a, b]$.

We note that the above theorem is given for statistical convergence, but it also stands for $A$-statistical convergence. To obtain our main result we need the next lemma. 
Lemma 1. Let $A:=\left(a_{k n}\right)_{k, n \in \mathbb{N}}$ be a non-negative regular summability matrix and let the operators $\widetilde{L}_{n, \lambda}$ be defined by (2.12) such that the following conditions hold

(1) the sequence $\left(\left\|\widetilde{\psi}_{n}\right\|\right)_{n \in \mathbb{N}}$ is bounded,

(2) $\mathrm{st}_{A}-\lim _{n}\left\|\widetilde{\psi}_{n}\right\|=0$.

Then the following identities hold:

$$
\mathrm{st}_{A}-\lim _{n}\left\|\tilde{L}_{n, \lambda} e_{i}-e_{i}\right\|=0 \quad \text { for } i=0,1,2 .
$$

Proof. From (2.12) and (2.3) it follows that

$$
\left(\tilde{L}_{n, \lambda} e_{0}\right)(x)=\sum_{j \in I_{n}} u_{n, j}(\lambda(x)), \quad x \in[0, b] .
$$

Since $e_{0}(\lambda(x))=e_{0}(x)=1$ for all $x \in[0, b]$, we obtain

$$
\left|\left(\tilde{L}_{n, \lambda} e_{0}\right)(x)-e_{0}(x)\right|=\left|\sum_{j \in I_{n}} u_{n, j}(\lambda(x))-e_{0}(x)\right| \leq\left\|\sum_{j \in I_{n}} u_{n, j}-e_{0}\right\| .
$$

Now using the above relation and (2.7), we get (3.1) for $i=0$.

By the definition (2.12) of the operator $\widetilde{L}_{n, \lambda}$ we have

$$
\begin{aligned}
\left(\tilde{L}_{n, \lambda} e_{1}\right)(x)= & \sum_{j \in I_{n}} u_{n, j}(\lambda(x)) x_{n, j} \sum_{s \in J_{j}} v_{j, s}(x) y_{j, s} \\
& +x \sum_{j \in I_{n}} u_{n, j}(\lambda(x)) \sum_{s \in J_{j}} v_{j, s}(x) \\
& -x \sum_{j \in I_{n}} u_{n, j}(\lambda(x)) x_{n, j} \sum_{s \in J_{j}} v_{j, s}(x) .
\end{aligned}
$$

Using (2.3) and (2.4) we obtain

$$
\left(\widetilde{L}_{n, \lambda} e_{1}\right)(x)=x \sum_{j \in I_{n}} u_{n, j}(\lambda(x)), \quad x \in[0, b] .
$$

Hence, we get

$$
\begin{aligned}
\left|\left(\tilde{L}_{n, \lambda} e_{1}\right)(x)-e_{1}(x)\right| & =\left|x \sum_{j \in I_{n}} u_{n, j}(\lambda(x))-e_{1}(x)\right| \\
& =|x|\left|\sum_{j \in I_{n}} u_{n, j}(\lambda(x))-e_{0}(x)\right| \leq b\left\|\sum_{j \in I_{n}} u_{n, j}-e_{0}\right\| .
\end{aligned}
$$

Since for a given $\varepsilon>0$ we have

$$
T_{1}:=\left\{n \in \mathbb{N}:\left\|\tilde{L}_{n, \lambda} e_{1}-e_{1}\right\| \geq \varepsilon\right\} \subseteq\left\{n \in \mathbb{N}:\left\|\sum_{j \in I_{n}} u_{n, j}-e_{0}\right\| \geq \frac{\varepsilon}{b}\right\}:=T_{2},
$$


we get

$$
\sum_{T_{1}} a_{k n} \leq \sum_{T_{2}} a_{k n}
$$

Taking $k \rightarrow \infty$, we obtain (3.1) for $i=1$.

By (2.3)-(2.5), (2.11), and an elementary calculus it follows that

$$
\begin{gathered}
\left(\tilde{L}_{n, \lambda} e_{2}\right)(x)=\sum_{j \in I_{n}} u_{n, j}(\lambda(x)) x_{n, j}^{2} \psi_{j}(x)+x^{2} \sum_{j \in I_{n}} u_{n, j}(\lambda(x)) \\
=\sum_{j \in I_{n}} u_{n, j}(\lambda(x)) x_{n, j} \tilde{\psi}_{n}(x)+x^{2} \sum_{j \in I_{n}} u_{n, j}(\lambda(x)) .
\end{gathered}
$$

Let $M:=\sup _{n \in \mathbb{N}}\left\{\left\|\widetilde{\psi}_{n}\right\|\right\}$. Then

$$
\begin{aligned}
& \left|\left(\tilde{L}_{n, \lambda} e_{2}\right)(x)-e_{2}(x)\right| \leq\left|\widetilde{\psi}_{n}(x)\left(\sum_{j \in I_{n}} u_{n, j}(\lambda(x)) x_{n, j}-e_{1}(\lambda(x))\right)\right| \\
& +\left|\lambda(x) \widetilde{\psi}_{n}(x)\right|+\left|x^{2}\left(\sum_{j \in I_{n}} u_{n, j}(\lambda(x))-e_{0}(x)\right)\right| \\
& \leq M\left\|\sum_{j \in I_{n}} u_{n, j} x_{n, j}-e_{1}\right\|+b\left\|\tilde{\psi}_{n}\right\|+b^{2}\left\|\sum_{j \in I_{n}} u_{n, j}-e_{0}\right\| .
\end{aligned}
$$

Let us set $K:=\max \left\{M, b^{2}\right\}$. Then

$$
\left\|\tilde{L}_{n, \lambda} e_{2}-e_{2}\right\| \leq K\left(\left\|\sum_{j \in I_{n}} u_{n, j} x_{n, j}-e_{1}\right\|+\left\|\tilde{\psi}_{n}\right\|+\left\|\sum_{j \in I_{n}} u_{n, j}-e_{0}\right\|\right) .
$$

For a given $\varepsilon>0$, we put

$$
\begin{aligned}
U: & :=\left\{n \in \mathbb{N}:\left\|\sum_{j \in I_{n}} u_{n, j} x_{n, j}-e_{1}\right\|+\left\|\tilde{\psi}_{n}\right\|+\left\|\sum_{j \in I_{n}} u_{n, j}-e_{0}\right\| \geq \frac{\varepsilon}{K}\right\}, \\
U_{1} & :=\left\{n \in \mathbb{N}:\left\|\sum_{j \in I_{n}} u_{n, j} x_{n, j}-e_{1}\right\| \geq \frac{\varepsilon}{3 K}\right\}, \\
U_{2} & :=\left\{n \in \mathbb{N}:\left\|\widetilde{\psi}_{n}\right\| \geq \frac{\varepsilon}{3 K}\right\},
\end{aligned}
$$

and

$$
U_{3}:=\left\{n \in \mathbb{N}:\left\|\sum_{j \in I_{n}} u_{n, j}-e_{0}\right\| \geq \frac{\varepsilon}{3 K}\right\} .
$$

It is obvious that $U \subset U_{1} \cup U_{2} \cup U_{3}$. Inequality (3.2) implies that

$$
\sum_{n:\left\|\widetilde{L}_{n, \lambda} e_{2}-e_{2}\right\| \geq \varepsilon} a_{k n} \leq \sum_{n \in U} a_{k n} \leq \sum_{n \in U_{1}} a_{k n}+\sum_{n \in U_{2}} a_{k n}+\sum_{n \in U_{3}} a_{k n} .
$$


Passing to the limit as $k \rightarrow \infty$, we complete the proof.

Remark 1 . If the non-negative regular summability matrix $A$ is the Cesáro matrix of order one, then the $A$-statistical convergence reduces to the statistical convergence $[5,7]$. Consequently, Lemma 1 also stands for statistical convergence.

Using the above lemma and Theorem A, we obtain the following result.

Theorem 1. Let $A:=\left(a_{k n}\right)_{k, n \in \mathbb{N}}$ be a non-negative regular summability matrix and let the operators $\widetilde{L}_{n, \lambda}$ be defined by (2.12). If the conditions of Lemma 1 hold, then for any function $f \in C_{B}([0, b])$ we have

$$
\mathrm{st}_{A}-\lim _{n}\left\|\tilde{L}_{n, \lambda} f-f\right\|=0 .
$$

\section{EXAMPLE}

In what follows we present a particular sequence of positive linear operators of the form (2.12) which is statistically convergent with respect to the sup-norm to the approximated function but it is not uniformly convergent.

Let

$$
\tilde{l}_{n}:\left\langle[0,1],\{0, \ldots, n\}, \frac{j}{n}, u_{n, j}(x)\right\rangle, \quad(n, j) \in \mathbb{N} \times\{0, \ldots, n\}, x \in[0,1]
$$

be such that

$$
u_{n, j}(x)=\mathrm{C}_{n}^{j}\left(\phi_{n}(x)\right)^{j}\left(1-\phi_{n}(x)\right)^{n-j}, \quad x \in[0,1],
$$

and

$$
\phi_{n}= \begin{cases}e_{1} & \text { if } \lg n \notin \mathbb{N}_{0} \\ n e_{1} & \text { if } \lg n \in \mathbb{N}_{0}\end{cases}
$$

for all $(n, j) \in \mathbb{N} \times\{0, \ldots, n\}$. It is clear that taking $\phi_{n}=e_{1}$ for all $n \in \mathbb{N}, \tilde{l}_{n}$ becomes the $n$th Bernstein polynomial.

We must should now check that $\left(u_{n, j}\right)$ satisfies conditions (2.2), (2.7)-(2.9). It is obvious that (2.2) and (2.7) are fulfilled. By using an elementary calculus, we obtain

$$
\left|\sum_{j=0}^{n} u_{n, j}(x) \frac{j}{n}-e_{1}(x)\right|=\left|\phi_{n}(x)-e_{1}(x)\right|=0
$$

for all $n$ with the property $\lg n \notin \mathbb{N}_{0}$. Letting $\varepsilon>0$ we obtain

$$
\left\{n \in \mathbb{N}:\left\|\sum_{j=0}^{n} u_{n, j} \frac{j}{n}-e_{1}\right\| \geq \varepsilon\right\}=\left\{n \in \mathbb{N}: \lg n \in \mathbb{N}_{0}\right\},
$$

and (2.8) is thus satisfied.

It remains only to verify (2.9). Indeed, 


$$
\begin{aligned}
\left|\sum_{j=0}^{n} u_{n, j}(x)\left(\frac{j}{n}\right)^{2}-e_{2}(x)\right|=\mid \frac{\phi_{n}(x)}{n}+\frac{n-1}{n} & \left(\phi_{n}(x)\right)^{2}-e_{2}(x) \mid \\
= & \left|\frac{x}{n}+\frac{n-1}{n} x^{2}-x^{2}\right| \leq \frac{x}{n} \leq \frac{1}{n}
\end{aligned}
$$

for all $n$ with the property $\lg n \notin \mathbb{N}_{0}$. Letting $\varepsilon>0$ we get

$$
\left\{n \in \mathbb{N}:\left\|\sum_{j=0}^{n} u_{n, j}\left(\frac{j}{n}\right)^{2}-e_{2}\right\| \geq \varepsilon\right\} \subseteq\left\{n \in \mathbb{N}: \lg n \in \mathbb{N}_{0} \text { and } \frac{1}{n} \geq \varepsilon\right\}
$$

and, therefore, (2.9) is also satisfied.

Choosing

$$
l_{n}=s_{n}:\left\langle[0, \infty), \mathbb{N}_{0}, \frac{j}{n}, e^{-n x} \frac{(n x)^{j}}{j !} ; \frac{e_{1}}{n}\right\rangle, \quad(n, j) \in \mathbb{N} \times \mathbb{N}_{0}, x \in[0, \infty)
$$

(the Favard-Szász-Mirakjan operators), we are able to define the operators $\widetilde{L}_{n, \lambda}$ by putting

$$
\begin{aligned}
\left(\tilde{L}_{n, \lambda} f\right)(x)=\sum_{j=0}^{n} \sum_{s=0}^{\infty} \mathrm{C}_{n}^{j}\left(\phi_{n}(\lambda(x))\right)^{j}( & \left.-\phi_{n}(\lambda(x))\right)^{n-j} \\
& \times e^{-j x} \frac{(j x)^{s}}{s} f\left(\frac{s}{n}+\left(1-\frac{j}{n}\right) x\right)
\end{aligned}
$$

for all $x \geq 0, f \in C_{B}\left(\mathbb{R}_{+}\right)$, and $n \in \mathbb{N}$, where $\lambda:[0, \infty) \rightarrow[0,1]$ is a continuous function.

On the basis of Lemma 1 and Theorem 1 we deduce that the sequence of operators ( $\tilde{L}_{n, \lambda} f$ ) defined by (4.1) is $A$-statistically convergent to $f$ for any function $f \in$ $C_{B}(D)$, where $D$ is a compact interval on the positive semiaxis.

\section{REFERENCES}

[1] O. Agratini, "On some new operators of discrete type," in Proceedings of the Fourth International Conference on Functional Analysis and Approximation Theory, Vol. I (Potenza, 2000), no. 68, part I, 2002, pp. 229-243.

[2] O. Duman, M. K. Khan, and C. Orhan, " $A$-statistical convergence of approximating operators," Math. Inequal. Appl., vol. 6, no. 4, pp. 689-699, 2003.

[3] O. Duman and C. Orhan, "Statistical approximation by positive linear operators," Studia Math., vol. 161, no. 2, pp. 187-197, 2004.

[4] E. Erkuş, O. Duman, and H. M. Srivastava, "Statistical approximation of certain positive linear operators constructed by means of the Chan-Chyan-Srivastava polynomials," Appl. Math. Comput., vol. 182, no. 1, pp. 213-222, 2006.

[5] H. Fast, "Sur la convergence statistique," Colloquium Math., vol. 2, pp. 241-244 (1952), 1951.

[6] A. R. Freedman and J. J. Sember, "Densities and summability," Pacific J. Math., vol. 95, no. 2, pp. 293-305, 1981.

[7] J. A. Fridy, "On statistical convergence," Analysis, vol. 5, no. 4, pp. 301-313, 1985. 
[8] A. D. Gadjiev and C. Orhan, "Some approximation theorems via statistical convergence," Rocky Mountain J. Math., vol. 32, no. 1, pp. 129-138, 2002.

Author's address

C. Radu

Babeş-Bolyai University, Faculty of Mathematics and Computer Science, 1 Kogălniceanu St., 400084 Cluj-Napoca, Romania

E-mail address: rcristina@math.ubbcluj.ro 